\title{
Results from the EPOXI and StardustNExT Missions - A Changing View of Comet Volatiles and Activity
}

\author{
Karen Meech ${ }^{1}$, Michael F. A'Hearn ${ }^{2}$ and Joseph Veverka ${ }^{3}$ \\ ${ }^{1}$ Institute for Astronomy, Univ. of Hawaii, NASA Astrobiology Institute \\ email: meech@ifa.hawaii.edu \\ ${ }^{2}$ University of Maryland, ${ }^{3}$ Cornell University, USA
}

\begin{abstract}
Within a period of $\sim 3$ months there were two extended mission flybys of comets. Both encounters have provided an exciting new view of comet activity and volatile composition that is changing our paradigm of these small early solar system remnants. The EPOXI mission flew past the nucleus of comet 103P/Hartley 2 on 4 Nov. 2010. This small nucleus was known to be exceptionally active prior to the encounter, by virtue of a very large water production rate relative to its surface area. Both the encounter and ground-based data showed that comet Hartley 2 fs perihelion activity was dominated by sub-surface $\mathrm{CO} 2$ outgassing rather than by water, suggesting our classic comet formation picture is not correct. The gas flow carried large grains (up to $>10 \mathrm{~cm}$ in diameter) from the nucleus, and the icy grains contributed to the large observed water production. The $\mathrm{CO} 2$ abundance relative to water varies with rotation between $10-20 \%$ between the two lobes of the nucleus. The bi-lobed nucleus is rotating in an excited state, with a period that varied rapidly from $\sim 16.5 \mathrm{hrs}$ to longer than $18.5 \mathrm{hrs}$ over 3 months. The nucleus morphology was different from that of other nuclei visited by space craft, with some regions of rough topography in which surface ice was visible. On 2011 Feb. 14 the Stardust-NExT spacecraft flew past the nucleus of comet 9P/Tempel 1, the target of the Deep Impact (DI) experiment in July 2005. The mission goal was to look at the nucleus after and intervening perihelion passage, extending the surface area imaged during the DI encounter and also image the 2005 impact site. The layering seen during the DI flyby was exhibited over the areas newly imaged in the NExT flyby, and it was found that $30 \%$ of the nucleus was covered by smooth deposits that were likely caused by eruption of subsurface materials. Although it has long been known that comets lose on average $\sim$ a meter of their surface per perihelion passage, it was surprising to see that in the regions imaged by both DI and NExT there was little change in the surface photometric properties and morphology with the exception of the prominent smooth flow edges. As seen from both the spacecraft and ground-based campaign, the comet continued its trend of decreasing activity from previous perihelion passages. We will present highlights from both missions and discuss implications for formation scenarios.
\end{abstract}

